\title{
Exon-skipping drug pulls ahead in muscular dystrophy field
}

For Duchenne muscular dystrophy (DMD), the most common form of a group of musclewasting diseases, substantially effective treatments-never mind a cure-have long been elusive. But that finally may be changing: therapies tailored to correct the mutations in the dystrophin-encoding gene that cause the disorder are moving into late-stage development.

"We finally now have a handful of medicines aimed at repair or replacement of the primary genetic defect," says Carrie Miceli, co-director of the Center for Duchenne Muscular Dystrophy at the University of California-Los Angeles. "It's phenomenal that early trials [of these novel drugs] show even some improvement."

The recessive $\mathrm{X}$-linked disease occurs in about 1 in 3,600 male births. Boys lose the ability to walk at around age 12 and are plagued by respiratory problems in their teens; most die by their mid-20s. Corticosteroids, the current standard therapy, can prolong the ability to walk by a couple of years, but they have serious side effects including high blood pressure and weight gain. Now, a handful of new disease-modifying treatments are in phase 2 and 3 trials.

In July, Sarepta Therapeutics of Cambridge, Massachusetts stirred excitement with the announcement of results of a phase 2 trial of its exon-skipping drug eteplirsen. In DMD, when certain exons are mutated or deleted, the RNA is stalled by the fault, resulting in nonfunctional dystrophin protein. Antisense oligonucleotides bind the RNA and skip an exon next to a defective one, thereby correcting the reading frame and enabling the production of a shorter, but still functional, dystrophin protein. Eteplirsen, which uses an antisense oligonucleotide called phosphorodiamidate morpholino, targets exon 51 and could benefit about $13 \%$ of the total population with DMD.

In a study of 12 boys, aged 7 to 13 , clinical benefit was measured by the distance they walked in six minutes. The baseline for all the kids was around 400 meters. After just over eight months, the four boys who had received the highest dose of eteplirsen once weekly intravenously for the entire study walked about 9 meters fewer on average in the six-minute timeframe. By comparison, the four participants taking placebo for 24 weeks followed by 12 weeks of eteplirsen showed a 78-meter average decline from a baseline.

"This is really an initial step, but it produced a result that we really haven't seen with any other treatment," says Jerry Mendell, director of the Centers for Gene Therapy and Muscular Dystrophy at Nationwide Children's Hospital

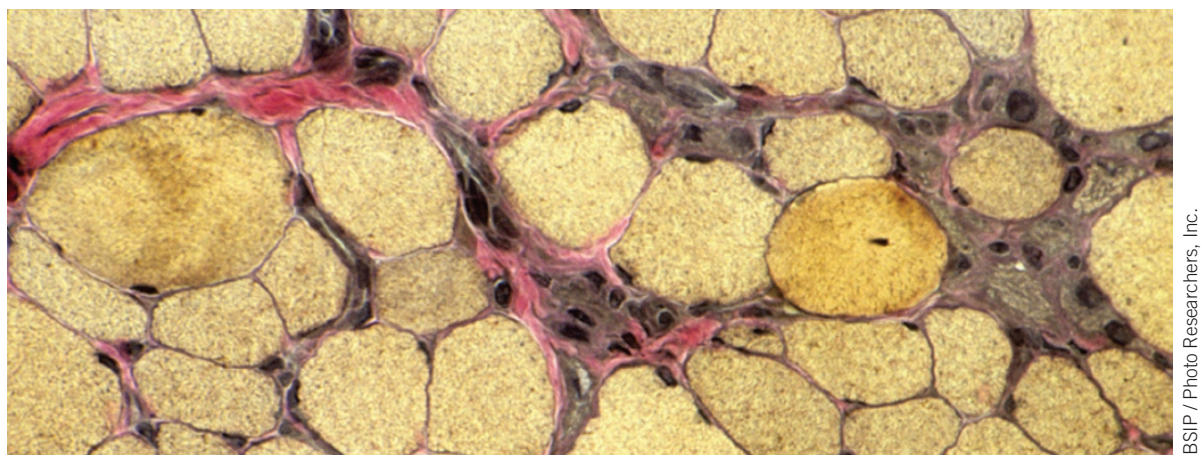

Muscling in on dystrophy: Drugs increase dystrophin levels in DMD-affected muscle tissue.

in Columbus, Ohio, and the study's principal investigator. "We achieved efficacy without any adversity — not a single side effect, which is pretty amazing for drug development."

In October, Sarepta, which recently changed its name from AVI BioPharma, plans to release results of a three-month extension study. It will reveal whether longer exposure to the drug improved gene expression and the patients' ability to walk. The thinking is, the more protein expression, the more improvement for the patient.

Sarepta does have competition, though. For example, an open-label 12-person study involving another exon-51-skipping drug known as drisapersen, which is being developed by the UK's GlaxoSmithKline in collaboration with the Dutch company Prosensa, actually showed a small but statistically significant average improvement in the six-minute walk test compared to baseline (N. Engl. J. Med. 364, 1513-1522, 2011). Results from a phase 3 trial launched last year are expected in 2014.

\section{A dose of reality}

One of the biggest challenges moving forward is dosing, says Mendell. "How much can you deliver, how much can get into the nucleus to overcome the normal biology of the gene defect, and will that produce a clinically meaningful result?"

Ideally, exon-skipping drugs of the future would turn patients with DMD into patients with an exceptionally mild form of the disease called Becker's muscular dystrophy by skipping exons 44 through 55 . That approach could treat more than half of boys with DMD, says Miceli, "but right now skipping that many exons isn't practical." Prosensa and Sarepta are both in the early stages of developing more drugs that aim forsingle skips in this region.

While researchers tackle those hurdles, another company is taking a different molecularbased approach. PTC Therapeutics's drug, ataluren, forces the translational readthrough of nonsense mutations in messenger RNAwhich make up about $13 \%$ of all DMD casesand allows production of functional dystrophin protein. The company hit a major snag in 2010 when its phase 2 trial results unexpectedly showed that a low dose, but not a high dose, was effective, as measured by the six-minute walk test.

To gain further information about the drug's safety and tolerability, the New Jerseybased company has since launched open-label multinational studies, enrolling participants from the phase 2 study and administering the lower dose. (The drug is also being tested in an ongoing phase 3 trial of people with a form of cystic fibrosis; see Nat. Med. 17, 396-397, 2011.) Currently, PTC is in discussions with regulatory agencies in the US and Europe to find a path forward for the drug in children with DMD, according to company president, Cláudia Hirawat.

"It's a good idea; it has promise, but whether they can achieve clinically meaningful results is really the issue at hand," says Mendell.

The two exon-skipping drugs and ataluren are farthest along in the pipeline, but other novel agents are in the preclinical or phase 1 stages. Mendell, for instance, is preparing a gene therapy clinical study to replace the entire gene. GlaxoSmithKline and St. Louis's SigmaAldrich are among the companies developing drugs that promote upregulation of utrophin, a cytoskeletal protein that showed promise as a dystrophin surrogate in animal studies. Other approaches include mysostatin inhibitors, which could increase muscle strength, and drugs that prevent fibrosis.

"Taking different approaches and treating different subgroups is a good thing," says Mendell. "It may be that multiple drugs and a number of approaches are needed for this very devastating disease."

Alisa Opar 